\title{
Breatherlike impurity modes in discrete nonlinear lattices
}

\author{
Hennig, D.; Rasmussen, Kim; Tsironis, G. P.; Gabriel, H.
}

Published in:

Physical Review E. Statistical, Nonlinear, and Soft Matter Physics

Link to article, DOI:

10.1103/PhysRevE.52.R4628

Publication date:

1995

Document Version

Publisher's PDF, also known as Version of record

Link back to DTU Orbit

Citation (APA):

Hennig, D., Rasmussen, K., Tsironis, G. P., \& Gabriel, H. (1995). Breatherlike impurity modes in discrete nonlinear lattices. Physical Review E. Statistical, Nonlinear, and Soft Matter Physics, 52(5), R4628-R4631. https://doi.org/10.1103/PhysRevE.52.R4628

\section{General rights}

Copyright and moral rights for the publications made accessible in the public portal are retained by the authors and/or other copyright owners and it is a condition of accessing publications that users recognise and abide by the legal requirements associated with these rights.

- Users may download and print one copy of any publication from the public portal for the purpose of private study or research.

- You may not further distribute the material or use it for any profit-making activity or commercial gain

- You may freely distribute the URL identifying the publication in the public portal

If you believe that this document breaches copyright please contact us providing details, and we will remove access to the work immediately and investigate your claim. 


\title{
Breatherlike impurity modes in discrete nonlinear lattices
}

\author{
D. Hennig, ${ }^{1,3}$ K. $\emptyset$. Rasmussen, ${ }^{2,3}$ G. P. Tsironis, ${ }^{3,4}$ and H. Gabriel ${ }^{1}$ \\ ${ }^{1}$ Fachbereich Physik, Institut für Theoretische Physik, Freie Universität Berlin, Arnimallee 14, 14195 Berlin, Germany \\ ${ }^{2}$ Institute of Mathematical Modelling, Technical University of Denmark, 2800 Lyngby, Denmark \\ ${ }^{3}$ Physics Department and Research Center of Crete, University of Crete, P.O Box 1527, Heraklion 71110, Crete, Greece \\ ${ }^{4}$ Center for Nonlinear Science and Department of Physics, University of North Texas, Denton, Texas 76203
}

(Received 14 June 1995)

\begin{abstract}
We investigate the properties of a disordered generalized discrete nonlinear Schrödinger equation, containing both diagonal and nondiagonal nonlinear terms. The equation models a linear host lattice doped with nonlinear impurities. We find different types of impurity states that form itinerant breather states in the vicinity of the impurities. We analyze the properties of these breathers analytically and numerically.
\end{abstract}

PACS number(s): 03.40.Kf, 42.65.Pc, 71.10. $+\mathrm{x}$

A rapidly growing number of recent investigations have been devoted to the combined effects of disorder and nonlinearity [1-5]. In infinite nonlinear lattices disorder is the source of two different types of phenomena. The first can be described as nonlinearity-induced symmetry breaking accompanied by the formation of an intrinsic local mode [3]. The second is met in cases, when disorder of the Anderson type is present in the nonlinear lattice $[4,5]$. In both cases disorder induces the formation of impurity states and dynamical features completely different from the ones in the corresponding linear lattices [6]. Some of the characteristic features of the nonlinear modes, such as exponentially localized shape and energies outside the linear lattice band [7-9], are quite similar to those of the (conventional) linear localized states. Others are completely different, e.g., the appearance of localized states only above a critical value of the nonlinearity strengths $[8,10]$. In this paper we shall describe a completely different type of nonlinear impurity state that is not characterized through a stationary shape and that emerges near impurity sites and forms an itinerant breather state in their vicinity. It is created through nonlinear impurities in the context of a generalized discrete nonlinear Schrödinger equation presenting a generic model of nonlinear lattice systems with disorder.

We are interested in the dynamics of quasiparticles (electrons or excitations) in a one-dimensional tight-binding linear lattice doped with impurities interacting nonlinearly with the host lattice. The dynamics is described by the following discrete nonlinear Schrödinger equation:

$$
\begin{aligned}
i \frac{d \psi_{n}(t)}{d t}= & \left(1+\mu\left|\psi_{n}\right|^{2} \delta_{n, l}\right)\left[\psi_{n+1}(t)+\psi_{n-1}(t)\right] \\
& -\gamma\left|\psi_{n}(t)\right|^{2} \psi_{n}(t) \delta_{n, l},
\end{aligned}
$$

where $\psi_{n}$ is a complex amplitude, and $\mu$ and $\gamma$ are real nonlinearity parameters corresponding to the nonlinear impurity-doped site situated at the site with index $l$ contained in the set of integers $\{m\}$. If $\{m\}$ is extended to cover the complete lattice, Eq. (1) becomes identical to an equation introduced by Salerno [11], studied initially by Cai, Bishop, and Gronbech-Jensen [12] and further in Refs. [13-15]. One notable feature of the equation studied by Cai et al. or its disordered version as given by Eq. (1) is that it interpolates between the conventional, nonintegrable, discrete nonlinear Schrödinger equation (DNLS) or discrete self-trapping equation (DST), $(\mu=0)$ and the completely integrable AblowitzLadik (AL) equation $(\gamma=0)$ [11-17]. The AL-DNLS equation (1) can be derived from the Hamiltonian

$$
\begin{aligned}
H= & -\sum_{n}\left\{\psi_{n}^{*} \psi_{n+1}+\psi_{n} \psi_{n+1}^{*}\right\} \\
& +\sum_{l \in\{m\}}\left\{\frac{\gamma}{\mu}\left|\psi_{l}\right|^{2}-\frac{\gamma}{\mu^{2}} \ln \left(1+\mu\left|\psi_{l}\right|^{2}\right)\right\}
\end{aligned}
$$

with a modified Poisson bracket given by $\left\{\psi_{n}, \psi_{m}^{*}\right\}$ $=i \delta_{n, m}\left(1+\mu\left|\psi_{l}\right|^{2} \delta_{n, l}\right), l \in\{m\}$ and $\dot{\psi}_{n}=\left\{H, \psi_{n}^{*}\right\}$ [12]. The diagonal DNLS-nonlinearity term modulates the on-site energies (taken to be equal to zero for simplicity) whereas the off-diagonal AL-nonlinearity affects the transfer matrix elements and hence the bandwidth. In addition to the energy $H$, the system of equations (1) possesses a further integral given by

$$
N=\sum_{n \in\{m\}}\left|\psi_{n}\right|^{2}+\sum_{l \in\{m\}} \frac{1}{\mu} \ln \left(1+\mu\left|\psi_{l}\right|^{2}\right) ;
$$

the latter serves as norm taken as $N=1$.

In order to investigate the character of localized solutions of Eq. (1) we first analyze the case of a single AL-impurity situated at the center site of the linear chain, i.e., $\{m\}=\{0\}$, where the total excitation energy is assumed to be localized initially. The initial amplitude is therefore given by $\left|\psi_{0}(t=0)\right|^{2}=[\exp (\mu)-1] / \mu$. We integrate the equations of motion numerically by using a Runge-Kutta method while checking the accuracy using the conserved quantities $H$ and $N$. Figure 1 shows the typical probability profile $\left|\psi_{n}(t)\right|^{2}$ at different lattice sites around the central site as a function of time for the parameter choice of $\mu=7$. We observe that part of the initial amplitude escapes to the linear chain sites with $|n|>1$, whereas the remaining amplitude is trapped in the initially excited central site and the two adjacent ones. For $\mu=7$ we have $\left|\psi_{1}\right|^{2}+\left|\psi_{-1}\right|^{2}+\ln \left(1+\mu\left|\psi_{0}\right|^{2}\right) / \mu \approx 0.67$. The phenomenon of the amplitude trapping in the vicinity of the impurity site was already observed in DNLS-like impurities 


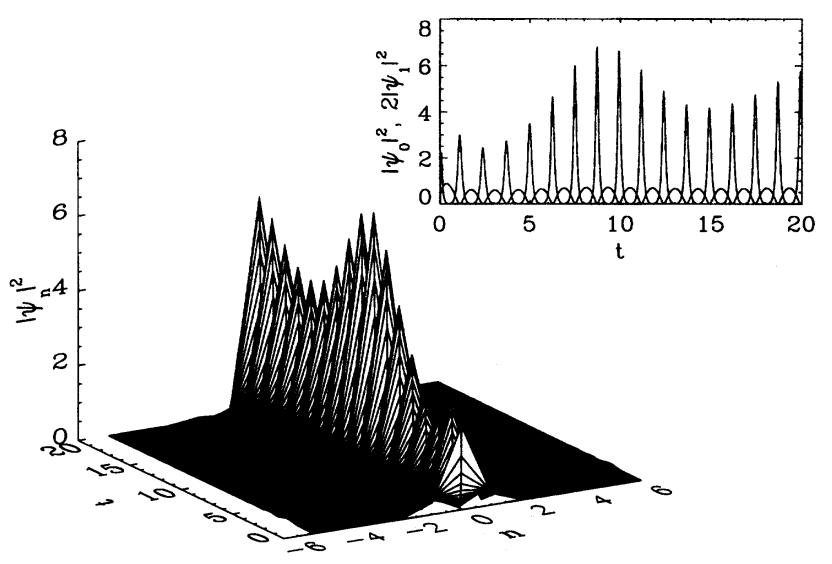

FIG. 1. Probability profile of the AL breather with $\mu=7$. The inset shows the time evolution of the squared central site amplitude and the out-of-phase oscillation of $2\left|\psi_{1}\right|^{2}$ at the adjacent sites.

studies for some nonlinearity parameter regimes [5-10]. However, in these nonlinear cases, the impurity state profile reaches a stationary exponentially localized shape [8]. In contrast we observe the surprising feature that the trapped state does not become stationary at all, but is characterized by an itinerant out-of-phase energy exchange between the central site and the two neighboring linear sites. Thus the produced localized impurity state is a long-lived stable breather $[18,19]$. Increasing the nonlinearity strength $\mu$ results in higher (initial) central-site amplitudes, shorter transient times, and faster stable breather formation. We observe that the AL term suppresses energy transfer from the initially excited central site to those beyond $|n| \geqslant 2$, an effect that is progressively enhanced with increasing $\mu$ and results in selftrapping of the excitation energy in the trimer segment, $n=-1,0,1$. Thus, for large $\mu$ values the central dynamical trimer segment effectively decouples from the rest of the chain. We mention that the breatherlike impurity state can also be formed by initial energy depositing in the linear part of the chain.

To gain more insight into the dynamics of the breather, we isolate the central three sites and study the resulting AL trimer. We make the ansatz $\psi_{0}(t)=A(t)$ and $\psi_{ \pm 1}(t)=i B(t)$ with real amplitudes $A$ and $B$. Its specific form is motivated by the fact that in the chain for a real initial condition $\psi_{0}(0)$, the solution alternates between purely real and imaginary amplitudes at different sites. After some straightforward algebra we obtain the integrable system of equations

$$
\dot{A}=2\left(1+\mu A^{2}\right) B, \quad \dot{B}=-A,
$$

which admits the following conserved integral (the norm for the truncated trimer)

$$
N=2 B^{2}+\frac{1}{\mu} \ln \left(1+\mu A^{2}\right) .
$$

In Fig. 2(a) we plot the level curves for $N=1$ in the $A-B$ plane for different $\mu$ values. We observe the enhanced "an-
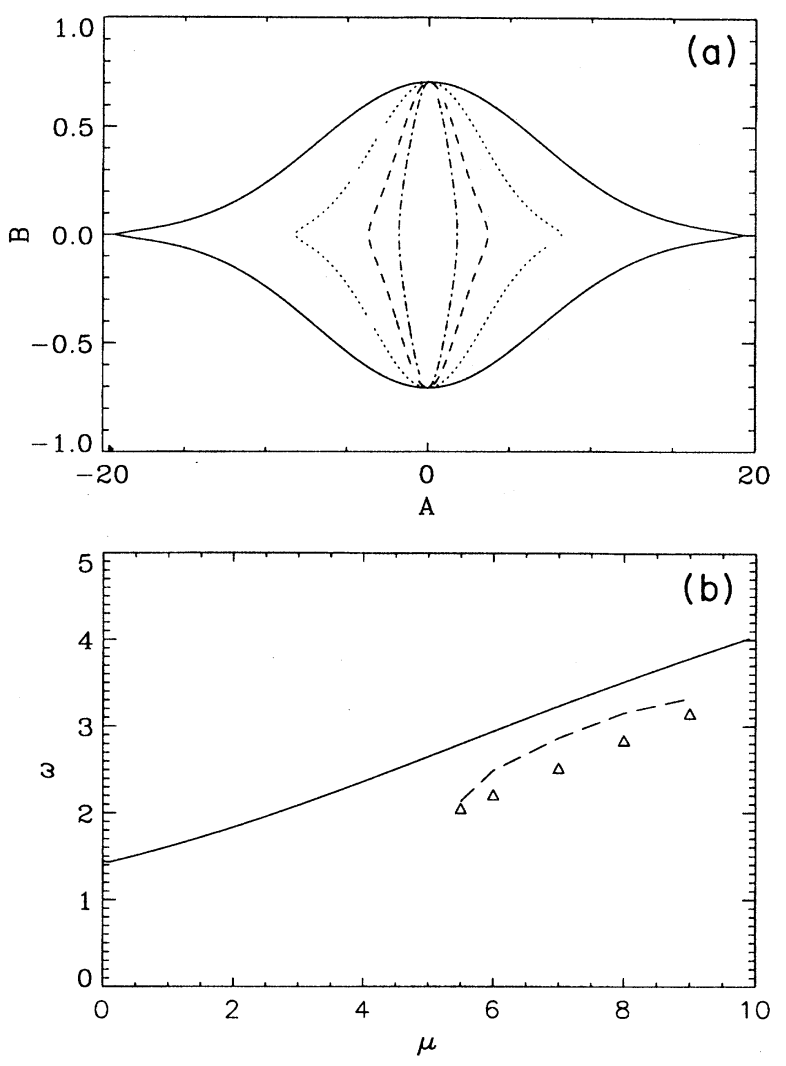

FIG. 2. (a) The level curves of $N=1$ in the $A-B$ phase plane of an $A L$ trimer determined by the equations in (4). We show the level curves of the norm $N$ for different $\mu$ values. Dashed-dotted line: $\mu=2$; Long-dashed line: $\mu=4$; short-dashed line: $\mu=6$; full line: $\mu=8$. (b) Oscillation frequency of the (genuine) $\mathrm{AL}$ trimer as a function of $\mu$ calculated through Eq. (6). The triangles correspond to the resonant oscillation frequency of the breathing mode in the lattice and are obtained numerically. The dashed lines represent the application of the expression of Eq. (6) for $N=N(\mu)$, where $N(\mu)$ has been numerically determined from the complete chain simulations. The agreement with the exact numerical result has been improved. Note that the true breather disappears when its frequency becomes identical to the upper band edge frequency.

harmonic" deformation of these curves with increasing $\mu$. The breather period for the energy exchange on the trimer sites is readily obtained as

$$
T_{t r}=2 \int_{-\sqrt{N / 2}}^{\sqrt{N / 2}} \frac{d B}{\sqrt{\frac{1}{\mu}}\left\{\exp \left[\mu\left(N-2 B^{2}\right)\right]-1\right\}} .
$$

Figure 2(b) shows the frequency of the energy exchange $\omega_{t r}=2 \pi / T_{t r}$ as a function of the nonlinearity strength $\mu$ (full line). The triangles correspond to the breather frequency obtained from numerical results for the infinite chain. We note that although the trimer result underestimates the actual frequency of the breather oscillation in the chain, it gives the correct slope as a function of $\mu$.

When two or more AL nonlinear impurities separated by a distance of at least three sites are placed in the lattice, a formation of a corresponding number of breather impurity 


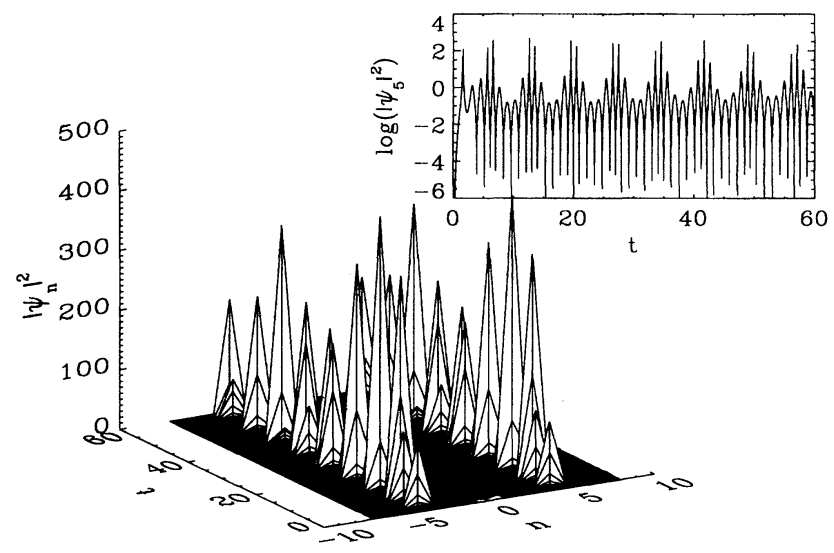

FIG. 3. Probability profile for the case of two AL impurities arranged at a distance of nine lattice sites apart from each other. The initial energy is placed in the center site between the two impurity sites. We observe the formation of AL breathers around each of the impurity sites. The inset shows the time evolution of the natural logarithm of the center amplitude of one of the breathers, where after a short transient period the regime of the stable itinerant oscillation is reached. [Due to the symmetry $\psi_{n}(t)=\psi_{-n}(t)$ it is sufficient to show only the amplitude $\left|\psi_{5}(t)\right|^{2}$.]

states is possible. As a result we can form a lattice of breathers each of which is created in the vicinity of an impurity and involves the two sites around it. In Fig. 3 we show the formation of two such breatherlike impurity states under the initial condition of depositing all the entire excitation energy in the linear site bisecting the distance between the nonlinear impurities.

Having investigated the properties of the AL-type impurities we now turn to the case of AL-DNLS impurities governed by Eq. (1). We note that pure DNLS impurities in a linear lattice chain provide a stationary self-trapped mode at the impurity site for $\gamma \geqslant 3.2[8,10]$ and that this localized state is well fitted by an exponential $[7,8]$. An interesting question remains to be answered: What will be the result of the competition between the dynamical self-trapped breathing mode supported by nondiagonal AL impurities and the stationary self-trapping mode originating from diagonal DNLS impurities? In Fig. 4 the probability profile for the combined case of $\mu=7$ and $\gamma=0.5$ is depicted. As can be seen from the inset, showing the time evolution of the amplitudes at the central site and the neighboring ones (periodic) oscillations of the amplitudes still exists. But compared to its pure AL counterpart $(\gamma=0)$ (see Fig. 1), for the com-

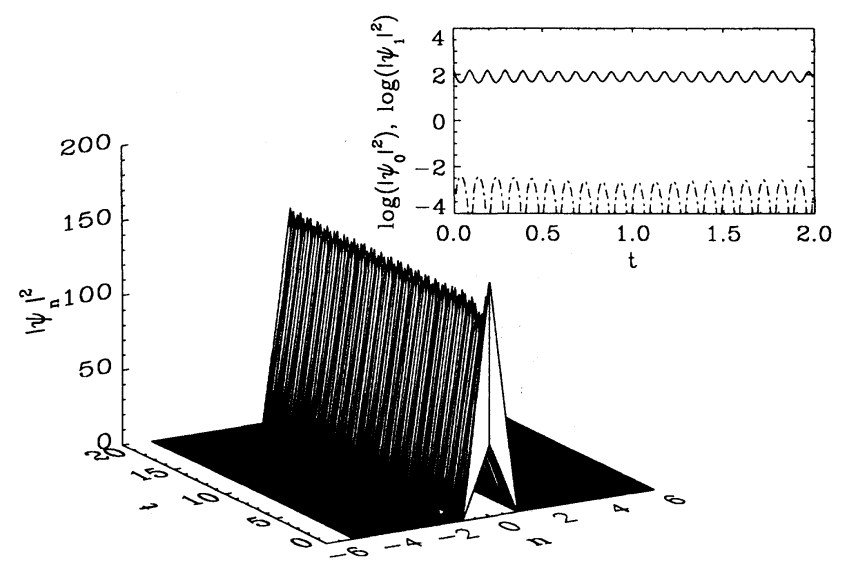

FIG. 4. Amplitude profile for the combined AL-DNLS impurity. Parameters are $\mu=7$ and $\gamma=0.5$. The time evolution of the natural logarithm of the central site amplitude as well as that of its neighboring ones presented in the inset illustrate the self-trapping of the excitation energy at the central DNLS site.

bined AL-DNLS impurity there is no complete energy exchange between the central site and its adjacent sites pointing to the bias of self-trapping at the initially excited nonlinear DNLS-impurity site. Indeed, with increasing $\gamma$ the energy transfer dynamics becomes more and more dominated by the DNLS term in the sense that although the out-of-phase oscillations are sustained, the amount of energy which remains in the central impurity site increases, whereas the maximal amplitudes at the adjacent sites diminish. There is almost no dynamical energy transfer in lattice sites apart from the central site, hence a self-trapped mode is created at the DNLS impurity site whose amplitude performs small oscillations around a mean value.

The existence of different types of impurity states in nonlinear models shows that disorder in nonlinear systems can lead to very different physical behaviors from the usual linear ones. The existence of genuine breathing modes in the AL-DNLS system can lead to the formation of a lattice of breathers each one of which is induced by the existence of a single impurity site. The formation of this lattice can have profound effects on the propagation properties of electrons in strongly interacting quasi-one-dimensional materials.

This work was supported by the Deutsche Forschungsgemeinschaft via Sonderforschungsbereich 337. Two of the authors (D.H.) and (K.Ø.R.) gratefully acknowledge support from Human Capital and Mobility Grant No. ERBCHRXCT930331 of the European Union and the warm hospitality of the Research Center of Crete, Greece.
[1] Disorder and Nonlinearity, edited by A.R. Bishop, D.K. Campbell, and S. Pnevmatikos (Springer-Verlag, New York, 1989).

[2] Disorder with Nonlinearity, edited by F. Abdullaev, A.R. Bishop, and S. Pnevmatikos (Springer-Verlag, Berlin, 1992).

[3] S. Takeno and S. Homma, J. Phys. Soc. Jpn. 60, 731 (1991),
62, 835 (1993); A.J. Sievers and S. Takeno, Phys. Rev. Lett. 61, 970 (1988); S. Takeno and K. Hori, J. Phys. Soc. Jpn. 60, 947 (1991); S. Takeno, ibid. 61, 2821 (1992); S.R. Bickham, S.A. Kiselev, and A.J. Sievers, Phys. Rev. B 47, 14206 (1993); J.B. Page, ibid. 41, 7835 (1990); K.W. Sandudsky, J.B. Page, and K.E. Schmidt, ibid. 46, 6161 (1992). 
[4] D. L. Shepelyansky, Phys. Rev. Lett. 70, 1787 (1993).

[5] M. I. Molina and G. P. Tsironis, Phys. Rev. Lett. 73, 464 (1994).

[6] E.N. Economou, Green's Functions in Quantum Physics, Springer Series in Solid State Sciences Vol. 7 (Springer-Verlag, Berlin, 1979).

[7] M.I. Molina and G.P. Tsironis, Phys. Rev. B 47, 15330 (1993).

[8] D. Chen, M. Molina and G.P. Tsironis, J. Phys. Cond. Matter 5, 8689 (1993).

[9] G.P. Tsironis, M.I. Molina, and D. Hennig, Phys. Rev. E 50, 2365 (1994).

[10] D. Dunlap, V.M. Kenkre, and P. Reineker, Phys. Rev. B 47, 14842 (1993).
[11] M. Salerno, Phys. Rev. A 46, 6856 (1992).

[12] D. Cai, A.R. Bishop, and N. Grønbech-Jensen, Phys. Rev. Lett. 72, 591 (1994).

[13] D. Cai, A.R. Bishop, N. Grønbech-Jensen, and M. Salerno, Phys. Rev. Lett. 74, 1186 (1995).

[14] Yu.S. Kivshar and M. Salerno, Phys. Rev. E 49, 3543 (1994).

[15] D. Hennig, N.G. Sun, H. Gabriel, and G.P. Tsironis, Phys. Rev. E 52, 255 (1995).

[16] M.J. Ablowitz and J.F. Ladik, J. Math. Phys. 17, 1011 (1976).

[17] J.C. Eilbeck, P.S. Lomdahl, and A.C. Scott, Physica 16, 318 (1985).

[18] S. Aubry, Physica D71, 196 (1994).

[19] R.S. MacKay and S. Aubry, Nonlinearity 7, 1623 (1994). 